\title{
Strong coupling between excitons in transition metal dichalcogenides and optical bound states in the continuum
}

\author{
K. L. Koshelev, ${ }^{1,2,{ }^{*}}$ S. K. Sychev, ${ }^{1}$ Z. F. Sadrieva, ${ }^{1}$ A. A. Bogdanov, ${ }^{1}$ and I. V. Iorsh ${ }^{1}$ \\ ${ }^{1}$ ITMO University, 197101 St. Petersburg, Russian Federation \\ ${ }^{2}$ Nonlinear Physics Centre, Australian National University, Canberra ACT 2601, Australia
}

(Received 21 February 2018; revised manuscript received 19 August 2018; published 17 October 2018)

\begin{abstract}
Being motivated by recent achievements in the rapidly developing fields of optical bound states in the continuum (BICs) and excitons in monolayers of transition metal dichalcogenides, we analyze strong coupling between $\mathrm{BICs}$ in $\mathrm{Ta}_{2} \mathrm{O}_{5}$ periodic photonic structures and excitons in $\mathrm{WSe}_{2}$ monolayers. We demonstrate that the giant radiative lifetime of a BIC allows us to engineer the exciton-polariton lifetime, enhancing it by two orders of magnitude compared to a bare exciton. We show that the maximal lifetime of a hybrid light-matter state can be achieved at any point of $\mathbf{k}$ space by shaping the geometry of the photonic structure. Our findings open a different route for the realization of moving exciton-polariton condensates with a nonresonant pump and without Bragg mirrors, which is of paramount importance for polaritonic devices.
\end{abstract}

DOI: 10.1103/PhysRevB.98.161113

\section{INTRODUCTION}

Monolayers of transition metal dichalcogenides (TMDCs) are a certain class of postgraphene two-dimensional materials [1], attracting vast research interest in recent years. TMDCs are direct-gap semiconductors, exhibiting strong light-matter coupling [2]. Moreover, these structures support excitons characterized by both large binding energies and sufficiently large Bohr radii [3], giving a rise to the existence of a strong excitonic response at room temperature and providing strong optical nonlinearity due to the excitonexciton interactions [4]. Another important property of the TMDC excitons is the large oscillator strength leading to the substantial exciton-photon interaction in these structures. These properties allow for the observation of the so-called strong coupling regime, leading to the emergence of exciton polaritons [5] at room temperatures in structures comprising a TMDC monolayer and an optical cavity.

Exciton polaritons have been extensively studied in the last two decades both due to their fascinating fundamental properties, such as high-temperature Bose condensation and superfluidity [6,7], and because of emerging applications such as virtually thresholdless polariton lasers [8] and energy effective all-optical logic gates [9]. Strong coupling of TMDC excitons with light was observed in structures resembling conventional microcavities, where the monolayer was sandwiched between two Bragg mirrors [10-13]. At the same time, since the fabrication of high-quality TMDC monolayers is based on mechanical exfoliation techniques [14], and thus not compatible with standard epitaxial techniques used for Bragg mirror fabrication, the realization of the structures considered in Refs. [10-13] is quite technologically demanding. It would be thus extremely useful to realize high-quality optical resonators without the requirement for the growth of an upper

*ki.koshelev@gmail.com mirror. As an alternative, a whispering gallery mode (WGM) in a disk resonator could be used for the realization of a strong coupling regime [15]. However, this approach also requires sophisticated fabrication techniques and precise positioning of the monolayer over the region of the disk resonator where the WGM mode has antinodes.

In this Rapid Communication we propose an alternative scheme for the realization of strong exciton-photon coupling in two-dimensional (2D) materials without using mirrors, which is beneficial both in terms of ease of realization and tunability. We focus on structures comprising a TMDC monolayer and a photonic crystal slab (PCS). The idea behind that is the exploitation of so-called optical bound states in the continuum (BICs) [16-18], supported by a PCS, which possess giant quality factors. BICs in periodic photonic structures originate because of the destructive interference of contrapropagating waveguide modes coupled via the periodic potential of a PCS. They are characterized by the Bloch vector lying within the light cone in vacuum and high radiative quality factors (up to $2 \times 10^{6}$ experimentally measured in photonic crystal slabs [19]). BICs in photonic crystal slabs and plasmonic lattices being high- $Q$ resonant modes have already found their applications in sensing [20], filtering [21], nonlinearly tunable devices [22], and lasing [23,24].

\section{MODEL}

The design of structure is shown in Fig. 1-the sample consists of a $\mathrm{WSe}_{2}$ flake placed on the top of a $\mathrm{Ta}_{2} \mathrm{O}_{5}$ PCS grating. We notice that strong light-matter coupling demands both high- $Q$ photonic structures and long-living excitonic states. We address this problem by adjusting the PCS geometrical and material parameters to the BIC regime which provides a giant quality factor of the resonator limited by absorption, surface roughness, and finite size of the sample [25].

We begin with an analysis of the eigenmode spectra of the PCS applying the guided-mode expansion (GME) method [26] widely used for the characterization of photonic 


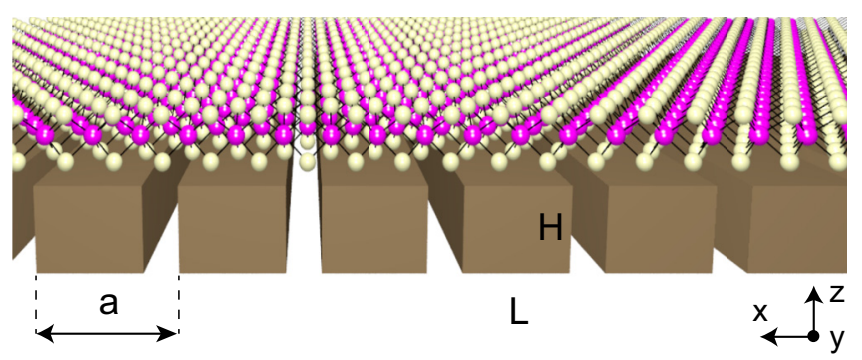

FIG. 1. An isometric view of a PCS-TMDC structure. The refractive index of $\mathrm{Ta}_{2} \mathrm{O}_{5}$ bars is equal to 2.1. The TMDC monolayer made of $\mathrm{WSe}_{2}$ is laid on top of the PCS.

eigenmodes of periodic photonic structures [27-29]. Within this approach radiative losses of leaky modes with frequencies above the light line are calculated by coupling coefficients to the continuum of scattering modes of the effective waveguide using the perturbation theory.

We consider an air-suspended one-dimensional $\mathrm{Ta}_{2} \mathrm{O}_{5}$ grating with air environment below and above it. The PCS consists of rectangular bars with a height of $H$ and a width of $L$ being spaced equidistant with a period of $a$ (see Fig. 1). We put the refractive index of $\mathrm{Ta}_{2} \mathrm{O}_{5}$ equal to 2.1 which is appropriate for the red band of the visible spectrum range [30]. The calculations are performed for the PCS with $a=1.03 \mathrm{H}$, $L=0.90 a$, and the eigenvalue problem is truncated by 101 plane waves and eight guided modes of an effective waveguide kept in the expansion [26].

The spectra of eigenfrequencies $\omega$ and inverse radiation lifetimes $\gamma=1 /\left(2 \tau_{\text {rad }}\right)$ of the PCS for in-plane wave vectors lying along the $x$ direction of the first Brillouin zone are shown in Figs. 2(a) and 2(c) for transverse electric (TE)polarized modes and in Figs. 2(b) and 2(d) for transverse magnetic (TM)-polarized modes, respectively. The dispersion curves under the light line $\omega=c k_{x}$ describe pure guided modes with zero diffraction losses while the photonic states above the light line are leaky since their radiation lifetime is finite. BICs represent unusual leaky modes with $\gamma=0$ and can be formed both at the center of the Brillouin zone (at- $\Gamma$ BIC) and at specific points between the zone edge and center (off- $\Gamma$ BIC) [31]. Importantly, both types of BICs are topologically stable against perturbations of the PCS geometry [31]. Therefore, while we do not account for the material dispersion and the PCS band structure scales linearly with the size parameters, the position of a BIC can be tuned, for example, by a variation of the structure height $H$.

The optical properties of $\mathrm{WSe}_{2}$ monolayers are governed by very robust excitons with binding energies of the order of $500 \mathrm{meV}$ [32]. We study A-type excitons representing bound states of electrons in the conduction band and holes in the upper subband of the valence band of the $\mathbf{K}_{+}$and $\mathbf{K}_{-}$valleys [33]. The optical selection rules result in the distinction of bright and dark excitons being active for in-plane and out-ofplane polarization of the incident light, respectively. We focus on bright excitonic states, which represent, in general, a pair of valley-degenerate states with $\sigma_{+}$and $\sigma_{-}$polarizations. In this case a linearly polarized pump excites a superposition of excitons with total polarization along the in-plane component of the electric field of light.
To investigate the light-matter interaction between excitons in the TMDC monolayer and BICs in the PCS we apply a full quantum formalism for both photonic and excitonic states which represents an extension of the GME method [34,35]. We start by the second quantization of the electromagnetic fields in a nonuniform dielectric environment and the excitonic wave function which exhibits properties of a gas of noninteracting bosons, while the density of excitons is much smaller than the saturation density. The coupling strength between photonic and excitonic modes is governed by the oscillator strength $f$ and the overlap integral of the mode profiles,

$$
V_{n, \mathbf{k}_{\|}}=-i f^{1 / 2} \int_{\text {cell }} d \mathbf{r}_{\|} \mathbf{e} \cdot \mathbf{E}_{n, \mathbf{k}_{\|}}^{\mathrm{up}}\left(\mathbf{r}_{\|}\right) e^{-i \mathbf{k}_{\text {exc }} \mathbf{r}_{\|}} .
$$

Here, $\mathbf{e}$ is the unit vector of exciton polarization, $\mathbf{k}_{\|}$and $\mathbf{k}_{\mathrm{exc}}$ are the in-plane wave vectors of the light and exciton, respectively, and $\mathbf{E}_{n, \mathbf{k}_{\|}}^{\mathrm{up}}$ is the electric field of the $n$th photonic mode at the upper surface of the PCS. Importantly, the exciton-photon interaction is allowed only for the wave vectors satisfying the condition $\mathbf{k}_{\mathrm{exc}}=\mathbf{k}_{\|}+\mathbf{G}$, where $\mathbf{G}$ is the reciprocal lattice vector. Finally, we formulate the total Hamiltonian and diagonalize it applying the generalized Hopfield transformation [36] which can be reduced to a non-Hermitian eigenvalue equation.

\section{STRONG LIGHT-MATTER COUPLING}

We apply the procedure for the characterization of the band structure and damping rates of exciton polaritons formed in the vicinity of the intersection between the BIC and the bare exciton dispersion curves. We consider cryogenic temperatures of about $4 \mathrm{~K}$ when the energy of an $A$-type bright exciton in $\mathrm{WSe}_{2}$ is $E_{\mathrm{exc}}=1.74 \mathrm{eV}$ [37]. The energy dispersion can be neglected at the scales of the problem and absorption losses are negligible because of low temperature. The radiative $\tau_{\mathrm{exc}}^{\mathrm{R}}$ and nonradiative $\tau_{\mathrm{exc}}^{\mathrm{NR}}$ lifetimes can be estimated as 1 and 3 ps, respectively [38]. For a bare exciton, the damping rate is mostly dominated by the radiative channel $\hbar /\left(2 \tau_{\text {exc }}\right)=$ $0.44 \mathrm{meV}$. However, when the TMDC is strongly coupled to the PCS, a new channel is enabled which represents the exchange of radiation between the exciton and the photonic system. This channel dominates over the direct radiation into free space [39]. It leads to the renormalization of the exciton lifetime which is used as a parameter for the eigenmode calculations. Therefore, in a strong coupling analysis we use the nonradiative lifetime $\hbar /\left(2 \tau_{\mathrm{exc}}^{\mathrm{NR}}\right)=0.11 \mathrm{meV}$ as the total exciton damping rate. The oscillator strength depends on $1 / \tau_{\mathrm{exc}}^{\mathrm{R}}$ and can be estimated as $f \simeq 6.5 \times 10^{-12} \mathrm{~cm} \mathrm{eV}^{2}$ (see Supplemental Material for details [40]). We focus on the off- $\Gamma$ BIC with TE polarization marked with a black square in Fig. 2(a) which provides sufficient coupling with the in-plane excitons according to Eq. (1). We tune the BIC frequency to a resonance with $E_{\text {exc }}$ by adjusting the height of the PCS to a value of $H=418.5 \mathrm{~nm}$. For numerical simulations we limit the basis to a linearly polarized in-plane exciton and the set of photonic modes used for the calculation of Fig. 2.

The spectra of energies $\hbar \omega$ and inverse lifetimes $\hbar /(2 \tau)$ of the upper and the lower exciton-polariton branches (UP and LP, respectively) calculated by means of the GME are 

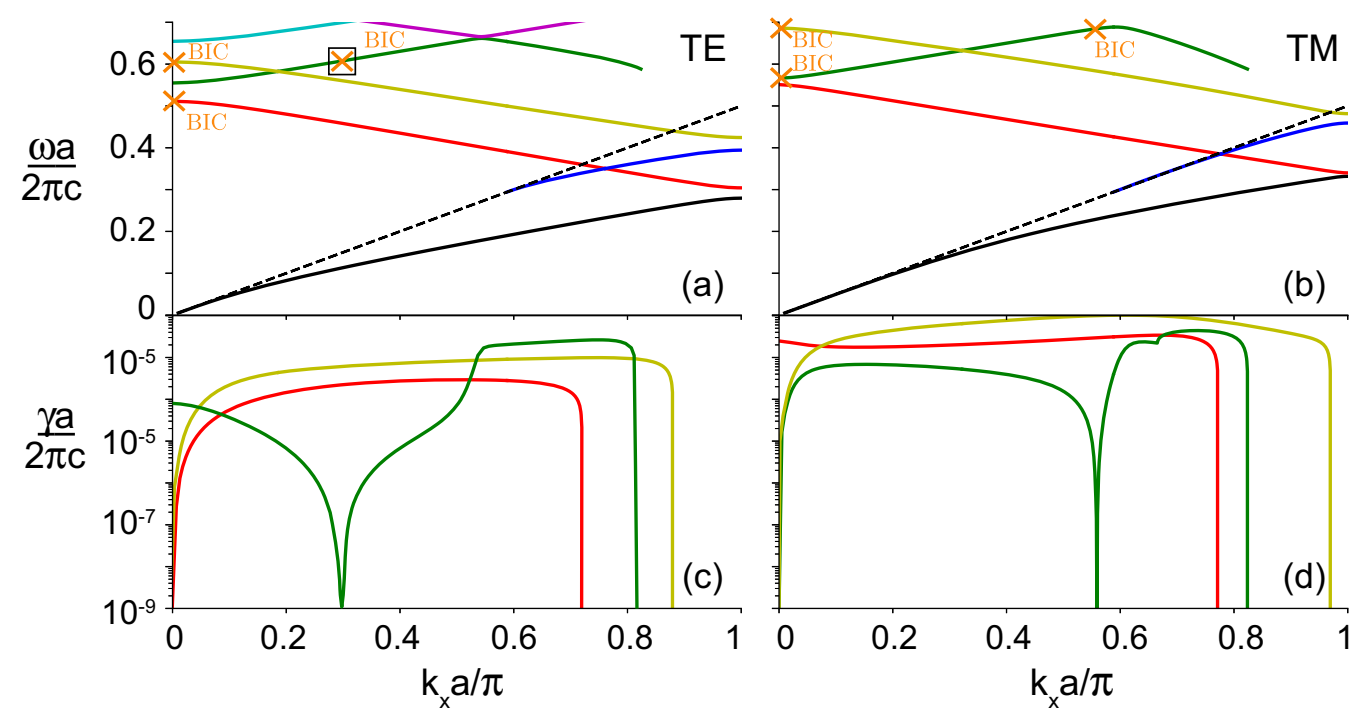

FIG. 2. Eigenmode spectrum of an air-suspended one-dimensional $\mathrm{Ta}_{2} \mathrm{O}_{5}$ grating. The band structure of the PCS with (a) TE-polarized and (b) TM-polarized modes, respectively. Black dashed lines represent the light lines $\omega=c k_{x}$. The spectrum of the inverse radiation lifetime for (c) TE-polarized and (d) TM-polarized modes, respectively. BICs are marked by orange crosses.

shown in Fig. 3 with solid lines. Figure 3(a) demonstrates strong coupling between the exciton and the off- $\Gamma$ BIC which manifests itself as an avoided resonance crossing with the Rabi splitting $2|V|$ equal to $5.7 \mathrm{meV}$. The radiation losses of both exciton-polariton branches are shown in Fig. 3(b) in comparison with the losses of pure photonic and excitonic modes. As it can be seen, for specific values of $k_{x}$ the lifetime of polariton modes can exceed the bare exciton lifetime by two orders of magnitude and reach a value of $75 \mathrm{ps}$. Such giant enhancement can be explained as a straightforward result of coupling of the exciton with a high- $Q$ photonic mode. As it was discussed before, the direct radiation of excitons into free space is strongly suppressed because of coupling to the PCS. At the same time BICs are entirely decoupled from the radiation continuum. The interplay between these effects leads to a strong suppression of all radiation mechanisms of exciton resulting in a giant enhancement of the polariton lifetime.

Most importantly, Fig. 3(b) shows that the maximal polariton lifetime can be realized at the point of phase space, where the group velocity of the mode is finite, which is not the center or the edge of the Brillouin zone. For the LP branch, this practically means that polaritons can form a macroscopically coherent long-lived state with nonzero energy flow at low temperatures, resulting in a propagating condensate. While usually a propagating condensate is realized by applying a resonant optical pump with a fixed in-plane wave vector, in the case of the off- $\Gamma$ BIC exploitation, the condensation to a propagating state can be achieved with a nonresonant pump (either an optical or electrical one).

Experimental methods of observation of strong lightmatter coupling include angle-resolved reflection spectroscopy. We model such an experiment by applying the Fourier modal method [45] with an adaptive spatial resolution [46] which is based on the expansion of the electromagnetic field and the permittivity function into a Fourier series. The reflectance is obtained via solving the wave equation in discretized Fourier space with proper boundary conditions. The increased efficiency is enabled by using the adaptive coordinates which allows us to achieve a $10^{-4}$ relative error by taking into account ten Fourier harmonics in the expansion. To obtain the peak positions and their linewidths we fit the reflectance spectrum to the Fano line shape using the Levenberg-Marquardt algorithm [47] (see Supplemental Material for details [40]). The real and imaginary parts of the eigenfrequency of the UP and the LP branches extracted by the fitting procedure are shown in Fig. 3 with open circles. We see that the GME method and the reflectance spectrum calculations show perfect agreement, providing the same value of the Rabi splitting and the 100-fold enhancement of the LP lifetime compared to a bare exciton.

We derive an analytical estimate for the maximal lifetime of the LP mode $\tau_{\mathrm{LP}}$ by using a simple two-level toy model which considers only one photonic and one exciton mode. While the Rabi splitting $2|V|$ [see Eq. (1)] is smaller than detuning $\Delta=\hbar \omega_{\mathrm{ph}}-\hbar \omega_{\mathrm{exc}}$, the degree of mixing between the photon and the exciton lifetimes is determined by the ratio $(|V| / \Delta)^{2}$. The interplay between the rapid increase of the photonic mode lifetime in the vicinity of the BIC and the enhancement of coupling to the exciton via a decrease of the detuning parameter results in the formation of an extremal point in the $\mathbf{k}$ space where the LP lifetime is maximal. Simple estimations (see Supplemental Material for details [40]) show that the LP lifetime is limited as

$$
\tau_{\mathrm{LP}} \leqslant \xi|V|^{-1}\left(\tau_{\mathrm{exc}}^{\mathrm{NR}}\right)^{1 / 2}
$$

where $\xi=1.66 \times 10^{-7} \mathrm{eV} \mathrm{s}^{1 / 2}$ is a constant determined by the PCS design. Equation (2) provides the estimate $\tau_{\mathrm{LP}} \leqslant$ $100 \mathrm{ps}$, which is in a good agreement with the numerical value of $75 \mathrm{ps}$.

Next, we calculate the dependence of the LP lifetime on the in-plane wave vector $\mathbf{k}=\left\{k_{x}, k_{y}\right\}$ within the 2D Brillouin zone using of the eigenmode analysis (see Fig. 4). One 


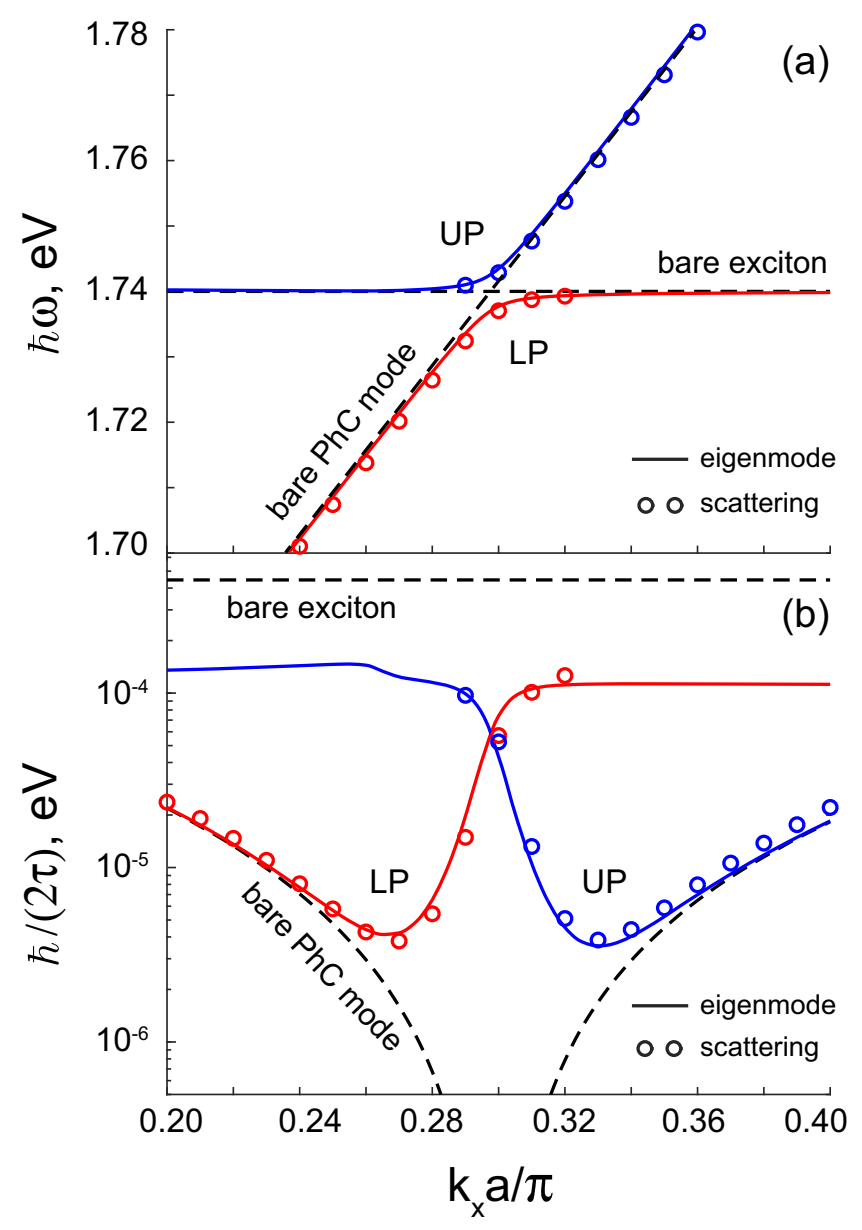

FIG. 3. Dispersion and inverse lifetime of exciton polaritons at the conditions of strong coupling between the TE-polarized photonic mode supporting an off- $\Gamma$ BIC and the in-plane polarized exciton with the energy of $1.74 \mathrm{eV}$ calculated by using the GME method (solid lines) and the fitting of the Fano line shape of reflection spectrum (open circles). The upper (UP) and lower (LP) polariton branches are shown with blue and red colors, respectively. The dispersion of a bare excitonic and a photonic mode is depicted with black dashed lines.

can see that the lifetime value exhibits a maximum at $\mathbf{k}=$ $\{ \pm 0.27 \pi / a, 0\}$ and decreases smoothly in the vicinity of these points. Importantly, the geometry of a one-dimensional PCS allows us to realize a BIC only on the $k_{y}=0$ or $k_{x}=0$ lines of the phase space. To enable control of the BIC position within the complete phase space of wave vectors, the broad class of two-dimensional periodic photonic structures can be used [31]. Therefore, the formation of long-living polariton states can be achieved for any value of $\mathbf{k}$, which leads to extreme flexibility in engineering polariton condensates with the desired value and direction of energy flow and even negative group velocities, which is of great interest for state-of-art applications [48].

Finally, we compare the prospects of our geometry with three conventional setups exploited for the observation of strong light-matter coupling in TMDCs [49,50]. In dielectric microcavities [10,13], metallic microcavities [51], and Tamm plasmon cavities [52], the typical value of the Rabi splitting is

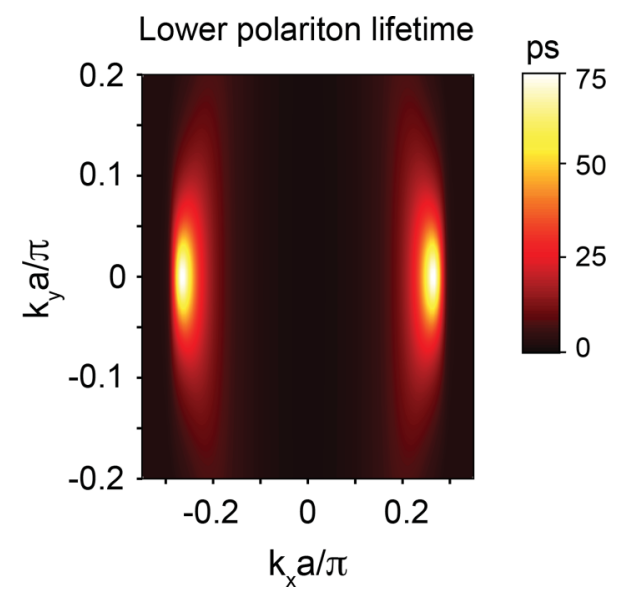

FIG. 4. The map of dependence of the lower polariton lifetime (red curve in Fig. 3) on the in-plane wave vector $\mathbf{k}$. The calculations are performed via the GME method.

between 15 and $100 \mathrm{meV}$ at room temperature. For plasmonic resonators $[53,54]$ this value can exceed $100 \mathrm{meV}$ because of the subwavelength localization of the electromagnetic energy. For dielectric gratings [55] at room-temperature conditions, the Rabi splitting is sufficiently lower, achieving values of order of 15-20 meV. While our system exhibits a slightly lower Rabi splitting, it has certain advantages over a microcavity design in terms of the fabrication simplicity. As to plasmonic resonators, despite the fact that these systems can exhibit a quite large Rabi splitting, they usually support only localized polaritons rather than propagating ones. Then, as compared to grating geometries, our setup demonstrates sufficiently larger quality factors. At last, the advantage of our setup is that it allows for excitonic lifetime engineering via coupling of an exciton to a BIC.

\section{CONCLUSION}

In conclusion, we have proposed an experimentally feasible scheme to achieve a strongly coupled exciton-photon system in a two-dimensional nanostructure comprising a TMDC monolayer and a periodic photonic nanostructure. Importantly, this scheme does not require the growth of Bragg mirrors, which simplifies the fabrication substantially. Moreover, we have shown that a giant 100 -fold enhancement of the polariton lifetime with a finite momentum can be achieved which allows for the nonresonant excitation of propagating polariton condensates. We believe these findings open different avenues for the applications of strong light-matter coupling at the nanoscale.

\section{ACKNOWLEDGMENTS}

Theoretical studies have been supported by the megagrant (No. 14.Y26.31.0015) and GosZadanie (No. 3.1365.2017/4.6). Simulations of the scattering spectrum have been supported by the Russian Science Foundation (No. 17-12-01581). K.L.K. acknowledges the Foundation for the Advancement of Theoretical Physics and Mathematics "BASIS" for the valuable financial support. 
[1] G. R. Bhimanapati, Z. Lin, V. Meunier, Y. Jung, J. Cha, S. Das, D. Xiao, Y. Son, M. S. Strano, V. R. Cooper et al., ACS Nano 9, 11509 (2015).

[2] K. F. Mak and J. Shan, Nat. Photonics 10, 216 (2016).

[3] G. Wang, A. Chernikov, M. M. Glazov, T. F. Heinz, X. Marie, T. Amand, and B. Urbaszek, Rev. Mod. Phys. 90, 021001 (2018).

[4] V. Shahnazaryan, I. Iorsh, I. Shelykh, and O. Kyriienko, Phys. Rev. B 96, 115409 (2017).

[5] A. V. Kavokin, J. J. Baumberg, G. Malpuech, and F. P. Laussy, Microcavities, Vol. 21 (Oxford University Press, Oxford, UK, 2007).

[6] J. Kasprzak, M. Richard, S. Kundermann, A. Baas, P. Jeambrun, J. Keeling, F. Marchetti, M. Szymańska, R. Andre, J. Staehli et al., Nature (London) 443, 409 (2006).

[7] A. Amo, J. Lefrère, S. Pigeon, C. Adrados, C. Ciuti, I. Carusotto, R. Houdré, E. Giacobino, and A. Bramati, Nat. Phys. 5, 805 (2009).

[8] P. Bhattacharya, T. Frost, S. Deshpande, M. Z. Baten, A. Hazari, and A. Das, Phys. Rev. Lett. 112, 236802 (2014).

[9] A. Amo, T. Liew, C. Adrados, R. Houdré, E. Giacobino, A. Kavokin, and A. Bramati, Nat. Photonics 4, 361 (2010).

[10] X. Liu, T. Galfsky, Z. Sun, F. Xia, E.-C. Lin, Y.-H. Lee, S. KénaCohen, and V. M. Menon, Nat. Photonics 9, 30 (2015).

[11] S. Dufferwiel, S. Schwarz, F. Withers, A. Trichet, F. Li, M. Sich, O. D. Pozo-Zamudio, C. Clark, A. Nalitov, D. Solnyshkov et al., Nat. Commun. 6, 8579 (2015).

[12] Z. Sun, J. Gu, A. Ghazaryan, Z. Shotan, C. R. Considine, M. Dollar, B. Chakraborty, X. Liu, P. Ghaemi, S. Kéna-Cohen et al., Nat. Photonics 11, 491 (2017).

[13] S. Dufferwiel, T. Lyons, D. Solnyshkov, A. Trichet, F. Withers, S. Schwarz, G. Malpuech, J. Smith, K. Novoselov, M. Skolnick et al., Nat. Photonics 11, 497 (2017).

[14] K. Novoselov, D. Jiang, F. Schedin, T. Booth, V. Khotkevich, S. Morozov, and A. Geim, Proc. Natl. Acad. Sci. USA 102, 10451 (2005).

[15] Y. Ye, Z. J. Wong, X. Lu, X. Ni, H. Zhu, X. Chen, Y. Wang, and X. Zhang, Nat. Photonics 9, 733 (2015).

[16] C. W. Hsu, B. Zhen, A. D. Stone, J. D. Joannopoulos, and M. Soljačić, Nat. Rev. Mater. 1, 16048 (2016).

[17] E. N. Bulgakov and A. F. Sadreev, Phys. Rev. B 78, 075105 (2008).

[18] S. G. Tikhodeev, A. L. Yablonskii, E. A. Muljarov, N. A. Gippius, and T. Ishihara, Phys. Rev. B 66, 045102 (2002).

[19] C. W. Hsu, B. Zhen, J. Lee, S.-L. Chua, S. G. Johnson, J. D. Joannopoulos, and M. Soljacic, Nature (London) 499, 188 (2013).

[20] A. A. Yanik, A. E. Cetin, M. Huang, A. Artar, S. H. Mousavi, A. Khanikaev, J. H. Connor, G. Shvets, and H. Altug, Proc. Natl. Acad. Sci. USA 108, 11784 (2011).

[21] J. M. Foley, S. M. Young, and J. D. Phillips, Phys. Rev. B 89, 165111 (2014).

[22] S. D. Krasikov, A. A. Bogdanov, and I. V. Iorsh, Phys. Rev. B 97, 224309 (2018).

[23] K. Hirose, Y. Liang, Y. Kurosaka, A. Watanabe, T. Sugiyama, and S. Noda, Nat. Photonics 8, 406 (2014).

[24] A. Kodigala, T. Lepetit, Q. Gu, B. Bahari, Y. Fainman, and B. Kanté, Nature (London) 541, 196 (2017).
[25] Z. F. Sadrieva, I. S. Sinev, K. L. Koshelev, A. Samusev, I. V. Iorsh, O. Takayama, R. Malureanu, A. A. Bogdanov, and A. V. Lavrinenko, ACS Photonics 4, 723 (2017).

[26] L. C. Andreani and D. Gerace, Phys. Rev. B 73, 235114 (2006).

[27] M. Galli, D. Gerace, K. Welna, T. F. Krauss, L. O'Faolain, G. Guizzetti, and L. C. Andreani, Opt. Express 18, 26613 (2010).

[28] M. Minkov, V. Savona, and D. Gerace, Appl. Phys. Lett. 111, 131104 (2017).

[29] J. P. Vasco and S. Hughes, Phys. Rev. B 95, 224202 (2017).

[30] T. J. Bright, J. Watjen, Z. Zhang, C. Muratore, A. A. Voevodin, D. Koukis, D. B. Tanner, and D. J. Arenas, J. Appl. Phys. 114, 083515 (2013).

[31] B. Zhen, C. W. Hsu, L. Lu, A. D. Stone, and M. Soljacic, Phys. Rev. Lett. 113, 257401 (2014).

[32] K. He, N. Kumar, L. Zhao, Z. Wang, K. F. Mak, H. Zhao, and J. Shan, Phys. Rev. Lett. 113, 026803 (2014).

[33] M. Glazov, E. Ivchenko, G. Wang, T. Amand, X. Marie, B. Urbaszek, and B. Liu, Phys. Status Solidi B 252, 2349 (2015).

[34] L. C. Andreani and D. Gerace, Phys. Status Solidi B 244, 3528 (2007).

[35] D. Gerace and L. C. Andreani, Phys. Rev. B 75, 235325 (2007).

[36] J. Hopfield, Phys. Rev. 112, 1555 (1958).

[37] G. Wang, C. Robert, A. Suslu, B. Chen, S. Yang, S. Alamdari, I. C. Gerber, T. Amand, X. Marie, S. Tongay et al., Nat. Commun. 6, 10110 (2015).

[38] M. Palummo, M. Bernardi, and J. C. Grossman, Nano Lett. 15, 2794 (2015).

[39] A. N. Poddubny, P. A. Belov, and Y. S. Kivshar, Phys. Rev. A 84, 023807 (2011).

[40] See Supplemental Material at http://link.aps.org/supplemental/ 10.1103/PhysRevB.98.161113 for details on the dependence of the oscillator strength on the exciton radiation lifetime, estimation of the maximal lifetime of the exciton-polariton mode using the two-level system approximation, calculation of the surface conductivity of a TMDC, and calculation of the mode volume of the BIC, which includes Refs. [41-44].

[41] E. L. Ivchenko, Optical Spectroscopy of Semiconductor Nanostructures (Alpha Science, Oxford, UK, 2005).

[42] L. Yuan and Y. Y. Lu, Phys. Rev. A 95, 023834 (2017).

[43] E. N. Bulgakov and D. N. Maksimov, Phys. Rev. Lett. 118, 267401 (2017).

[44] M. Merano, Phys. Rev. A 93, 013832 (2016).

[45] L. Li, J. Opt. Soc. Am. A 13, 1870 (1996).

[46] T. Vallius and M. Honkanen, Opt. Express 10, 24 (2002).

[47] M. V. Rybin, A. B. Khanikaev, M. Inoue, K. B. Samusev, M. J. Steel, G. Yushin, and M. F. Limonov, Phys. Rev. Lett. 103, 023901 (2009).

[48] K. L. Tsakmakidis, O. Hess, R. W. Boyd, and X. Zhang, Science 358, eaan5196 (2017).

[49] A. Krasnok, S. Lepeshov, and A. Alú, Opt. Express 26, 15972 (2018).

[50] C. Schneider, M. M. Glazov, T. Korn, S. Höfling, and B. Urbaszek, Nat. Commun. 9, 2695 (2018). 
[51] S. Wang, S. Li, T. Chervy, A. Shalabney, S. Azzini, E. Orgiu, J. A. Hutchison, C. Genet, P. Samor, and T. W. Ebbesen, Nano Lett. 16, 4368 (2016).

[52] N. Lundt, S. Klembt, E. Cherotchenko, S. Betzold, O. Iff, A. V. Nalitov, M. Klaas, C. P. Dietrich, A. V. Kavokin, S. Hofling et al., Nat. Commun. 7, 13328 (2016).
[53] J. Wen, H. Wang, W. Wang, Z. Deng, C. Zhuang, Y. Zhang, F. Liu, J. She, J. Chen, H. Chen et al., Nano Lett. 17, 4689 (2017).

[54] J. Cuadra, D. G. Baranov, M. Wersall, R. Verre, T. J. Antosiewicz, and T. Shegai, Nano Lett. 18, 1777 (2018).

[55] L. Zhang, R. Gogna, W. Burg, E. Tutuc, and H. Deng, Nat. Commun. 9, 713 (2018). 